\title{
An immunohistochemical approach to detect oncogenic CTNNB1 mutations in primary neoplastic tissues
}

\author{
Aytekin Akyol ${ }^{1,2,3,4} \cdot$ Günes Güner ${ }^{1,9} \cdot$ Havva Solak Özşeker $\mathbb{1}^{1,10} \cdot$ Aynur Işık $^{2} \cdot$ Özge Atci $^{5} \cdot$ Sarp Uzun ${ }^{6}$. \\ Emine Atayar $^{7} \cdot{\text { Fatih Ozaltin } \mathbb{D}^{7} \cdot \text { Gökhan Gedikoğlu }^{1} \cdot \text { Cenk Sökmensüer }}^{1} \cdot$ Eric R. Fearon $^{8}$
}

Received: 9 April 2018 / Revised: 17 July 2018 / Accepted: 3 August 2018 / Published online: 3 September 2018

(c) United States \& Canadian Academy of Pathology 2018

\begin{abstract}
The Wnt/ $\beta$-catenin signaling pathway is dysregulated in different types of neoplasms including colorectal cancer (CRC). Aberrant activation of this signaling pathway is a key early event in the development of colorectal neoplasms, and is mainly caused by loss of function mutations in Adenomatous Polyposis Coli (APC), and less frequently by $\beta$-catenin stabilization mutations via missense or interstitial genomic deletions in CTNNB1. In this study, we have defined an immunohistochemical algorithm to dissect Wnt pathway alterations in formalin-fixed and paraffin-embedded neoplastic tissues. Basically, consecutive sections of tumor specimens were stained by immunohistochemistry with two different monoclonal antibodies against $\beta$-catenin: one (anti-active $\beta$-catenin antibody) recognizes hypo-phosphorylated $\beta$-catenin and the other recognizes the total pool of $\beta$-catenin. We validated the strategy in the HCT116 CRC cell line which has an in-frame deletion of $\beta$ catenin serine 45, and then studied human tumor microarrays containing colon adenomas, CRCs, solid pseudopapillary neoplasms of the pancreas as well as the whole tissue sections of CRCs, desmoid fibromatosis, and pilomatrixoma of the skin. In some tumors, we found strong $\beta$-catenin cytoplasmic and/or nuclear staining with the total $\beta$-catenin antibody but no staining with the anti-active $\beta$-catenin antibody. This was inferred to be an altered/mutant $\beta$-catenin staining pattern. All six colon adenomas of the 126 total adenomas studied for the altered/mutant $\beta$-catenin staining pattern had presumptively pathogenic point mutations or deletions in CTNNB1. Four of $10 \mathrm{CRCs}$ with the alterated/mutant $\beta$-catenin staining pattern studied in depth, from 181 total CRCs from tissue microarray, had pathogenic CTNNB1 mutations. The frequencies of $C T N N B 1$ alterations in non-colonic tumors with altered/mutant $\beta$-catenin staining ranged between 46 and $100 \%$. Our results demonstrate that the immunohistochemical approach described here can detect oncogenic forms of $\beta$-catenin in primary tissue samples and can also highlight other tumors with presumptive novel defects activating the $\mathrm{Wnt} / \beta$-catenin pathway.
\end{abstract}

Electronic supplementary material The online version of this article (https://doi.org/10.1038/s41374-018-0121-9) contains supplementary material, which is available to authorized users.

Aytekin Akyol

aytekina@hacettepe.edu.tr

1 Department of Pathology, Hacettepe University Faculty of Medicine, Sihhiye, 06100 Ankara, Turkey

2 Transgenic Animal Technologies Research and Application Center, Hacettepe University, Sihhiye, 06100 Ankara, Turkey

3 Tumor Pathology Division, Cancer Institute, Hacettepe University, Sihhiye, 06100 Ankara, Turkey

4 Molecular Pathology Research and Application Center, Hacettepe University, Sihhiye, 06100 Ankara, Turkey

5 Biology Program, Department of Biology, Gazi University Faculty of Sciences, Ankara, Turkey

\section{Introduction}

Colorectal carcinomas (CRCs) are a major cause of cancerrelated mortality and morbidity in much of the world.

6 Basic Oncology Department, Tumor Biology and Immunology Ph. D. Program, Hacettepe University Cancer Institute, Sihhiye, 06100 Ankara, Turkey

7 Nephrogenetics Laboratory, Department of Pediatric Nephrology, Hacettepe University Faculty of Medicine, Sihhiye, 06100 Ankara, Turkey

8 Departments of Internal Medicine, Human Genetics, and Pathology, University of Michigan Medical School, Ann Arbor, MI 48109, USA

9 Present address: Mardin State Hospital, Mardin, Turkey

10 Present address: Training and Research Hospital, Muğla Sitk1 Koçman University, Muğla, Turkey 
Genetic and epigenetic alterations present in CRCs are being studied in depth and defects in various signaling pathways have been identified, including recurrent alterations in the $\mathrm{Wnt} / \beta$-catenin, transforming growth factor- $\beta$, receptor tyrosine kinase-RAS, phosphatidylinositol 3-phosphate, and P53 signaling pathways $[1,2]$. Genes encoding proteins in the Wnt/ $\beta$-catenin pathway are frequently targeted in CRCs, especially mutations in the $A P C$, which are present in about $80 \%$ of CRCs. In addition to CRC, the Wnt pathway is activated in neoplasms arising in tissues other than the colon and rectum [3, 4]. A gain-of-function mutation in the CTNNB1, which encodes for the $\beta$-catenin protein also causes aberrant Wnt pathway activation. The CTNNB1 cancer-associated mutations mostly affect the N-terminus of $\beta$-catenin, which contains conserved serine/threonine amino acid residues that are phosphorylated. The levels of the cytoplasmic pool of free $\beta$-catenin are tightly regulated by ubiquitination and proteasomal degradation [5, 6]. In normal physiological and pathological conditions, $\beta$-catenin's function in the Wnt signaling pathway is determined by its levels and subcellular localization. $\beta$-catenin in the nucleus functions as a transcription coactivator through binding to TCF/LEF transcription factor family members [7-9].

In recent years, mutation-specific monoclonal antibodies have been used in routine pathology diagnostics, mainly due to an increase in targeted therapy options for cancer patients [10]. Immunohistochemical methods for mutation determination provide an alternative to molecular methods, with both potential advantages and disadvantages. There are many different antibodies to detect mutant forms of proteins by immunohistochemistry in formalin-fixed paraffin-embedded (FFPE) cancer tissues. BRAF, KRAS, and p53 are examples of cancer-related proteins for which mutation-specific antibodies for immunohistochemistry are commercially available [11-13]. Here, we describe an immunohistochemical method that can be used to study Wnt pathway defects in primary neoplastic tissues.

\section{Materials and methods}

\section{Cell line and tissue block preparation}

The HCT 116 colon cancer cell line was kindly provided by Dr. Sreeparna Banerjee (Middle East Technical University, Ankara, Turkey) and routinely grown in media made with Dulbecco's Modified Eagle's Medium-F12 (DMEM-F12; Biowest, France) containing 10\% fetal bovine serum and penicillin/streptomycin. Cultured cells were harvested by scraping from the tissue culture dish and transferred into $50 \mathrm{ml}$ falcon tubes with the medium. Samples were briefly centrifuged for $5 \mathrm{~min}$ at $1670 \mathrm{rpm}$. The supernatant was discarded; $10 \mathrm{ml}$ of $96 \%$ ethanol was added into the cell pellet, mixed, and incubated for $30 \mathrm{~min}$; this fixation procedure was repeated twice. The tissue sample was wrapped with a paper towel, placed into tissue processing cassettes and put into $96 \%$ ethanol $-50 \%$ formaldehyde mixture (1:1) for $60 \mathrm{~min}$. Routine tissue processing, paraffin embedding, haematoxylin \& eosin, and immunohistochemical staining of unstained sections followed.

\section{Tissue samples and tissue microarray (TMA) construction}

Tumor samples accessioned at the Hacettepe University were obtained from the Pathology Department archives. A total of five TMAs were constructed from FFPE CRC and solid pseudopapillary tumor of pancreas tissues, using the manual Advanced Tissue Arrayer (ATA100, Chemicon). Two 1-mm spots of representative tumor tissues of 202 CRC cases of various histological subtypes (mostly adenocarcinoma, $n=193$ ) and 13 solid pseudopapillary tumors were included in these TMAs. Preneoplastic lesions of the colon and rectum were represented in four TMAs, comprising tissues from 139 polyps. One 3-mm diameter core per polyp case was included in these TMAs. The breakdown of the colon preneoplastic lesions included in these four TMAs were as follows: 21 tubulovillous adenomas, 24 traditional serrated adenomas, 77 tubular adenomas, 1 sessile serrated polyp, 3 serrated adenomas, and 13 other polyps ( 5 hyperplastic polyps, 5 hamartomatous polyps, 1 inflammatory polyp, and 1 inflammatory fibroid polyp). Desmoid fibromatosis $(n=6)$, pilomatrixomas $(n=9)$, and CRCs $(n=40)$ were not included in the TMAs; a whole section of one representative FFPE block was used for immunohistochemistry. The tissue studies were carried out with approval from the Non-Interventional Clinical Research Ethics Committee of Hacettepe University (TBK 09/7-45 and HEK 12/147-22).

\section{$\beta$-catenin immunohistochemistry and antibodies}

Two independent 4-micron-thick unstained sections from each tissue block were immunohistochemically stained for $\beta$-catenin, using two different antibodies. The mouse monoclonal antibody for $\beta$-catenin (BD Transduction Laboratories, BD Biosciences, San Jose, CA, Cat. No.: 610154, 1:1000) was used to show the total pool of $\beta$-catenin, and its subcellular localization (membranous, cytoplasmic, or nuclear). The other mouse monoclonal anti$\beta$-catenin antibody detects the so-called active form of $\beta$-catenin. The antibody was developed via immunization with an epitope that covers the first (N-terminal) 100 amino acid residues of $\beta$-catenin in its hypo/unphosphorylated state, especially when the T41 and S37 residues were not phosphorylated (Merck Millipore, Anti-Active- $\beta$-catenin 
[Anti-ABC] Antibody, clone 8E7, Cat. No.: 05-665, 1:100) $[14,15]$. Reproducibility of staining pattern with $\beta$-catenin antibodys' also confirmed in HCT116 colon cancer cell line tissue block and colon adenoma TMA slides by using NonPhospho (Active) $\beta$-catenin (Ser33/37/Thr41) (D13A1) Rabbit mAb (Cell Signalling) and total $\beta$-catenin mouse monoclonal antibody (Dako, Cat. No.: M3539). For immunohistochemical analysis of $\beta$-catenin, briefly, the unstained slides were de-paraffinized and incubated at $75^{\circ} \mathrm{C}$ for $40 \mathrm{~min}$ followed by two turns in xylene, $10 \mathrm{~min}$ each, and rehydrated gradually in decreasing concentrations of ethyl alcohol (96, 90, and 70\%, respectively). Endogenous peroxidase activity was blocked with $3 \% \mathrm{H}_{2} \mathrm{O}_{2}$ in methanol. Antigen retrieval was done by microwaving for $10 \mathrm{~min}$ in citrate buffer (pH 6.0) solution (Genemed Biotechnologies, Inc. South San Francisco, CA) for $\beta$-catenin and $30 \mathrm{~min}$ in 10 mM EDTA buffer, pH 8.0 (Dr. Zeydanl,, Ankara, Turkey) for active form of $\beta$-catenin (ABC). Slides were incubated with the primary antibodies for $1 \mathrm{~h}$ at room temperature. The UltraVision Polyvalent (Rabbit-Mouse) HRP Kit (TP-125-HL Thermo Scientific/LabVision), and the avidin-biotin peroxidase method was then used. The signals were developed using the chromogen, 3,3'-diaminobenzidine (DAB, TA-125-HD, Thermo Scientific/LabVision). Finally, samples were briefly counterstained with haematoxylin. The slides were dehydrated and prepared for microscopic examination. Sections stained with $\beta$-catenin and $\mathrm{ABC}$ were consecutively evaluated. The CRC TMA slides were scanned with Olympus VS 120 System and visualized via the OlyVIA software. Samples with Wnt pathway activation were defined by the presence of abundant cytoplasmic and nuclear accumulation of $\beta$-catenin. If the same tissue sample was negative for staining with the anti-ABC antibody, the finding was interpreted as evidence for an altered/mutant staining pattern for $\beta$-catenin.

\section{DNA isolation from FFPE tissue blocks}

Genomic DNA from FFPE colon adenoma, carcinoma, and non-colonic tumor samples were prepared with the microwave method, with slight modifications from a previously described protocol [16]. Specifically, two serial $6 \mu \mathrm{m}$ thick sections were generated-one section was used for $\mathrm{H} \& \mathrm{E}$ staining, and consecutive unstained section was used for DNA isolation. Neoplastic tissues were scraped from the unstained section with a sterile needle and transferred into an Eppendorf tube. Genomic DNA extraction was performed as was previously described, followed by ethanol precipitation. Finally, the DNA sample was diluted with $20 \mu$ of DNase RNase-free $\mathrm{H}_{2} \mathrm{O}$ and its concentration was measured with Nanodrop ND-2000 (Thermo Fisher) (concentrations were in the range of
20-90 ng/ $\mu \mathrm{l})$. The samples were then used for PCR amplification.

\section{RNA isolation and CDNA synthesis}

Total RNA extraction from the HCT 116 cell line and normal colon tissue were performed by using the TRIzol (Thermo Fisher) method. Total RNA from FFPE colon adenoma and carcinoma samples were extracted using the Qiagen RNeasy FFPE Kit as instructed in the vendors' manual. cDNA was synthesized using a high-capacity cDNA reverse transcription kit (Thermo Fisher) with random primers.

\section{Mutational analysis of CTNNB1}

Genomic DNA was extracted from FFPE samples of 6 colon adenomas, 10 CRCs, 6 desmoid fibromatosis, 5 pilomatrixomas, and 6 solid pseudopapillary tumors that exhibited an the altered/mutant $\beta$-catenin staining pattern, along with a HCT 116 tissue block and normal colon tissue. The DNA samples were used to amplify $C T N N B 1$ exon 3 (Primers; BCAT-F:5'-ATTTGATGGAGTTGGACATGGC-3' and BC AT-R:5'-CCAGCTACTTGTTCTTGAGTGAAGG-3') with PCR (polymerase chain reaction), which yielded a $226 \mathrm{bp}$ amplicon with the appropriate controls. For RT-PCR amplification of the exon 2-4 region of $C T N N B 1$, we used the cDNA samples from the same 6 colon adenoma and 10 CRC cases (Primers; CTNNB1-S:5'-CCTGAGGGTATTTGAAG TATAC- $3^{\prime}$ and CTNNB1-AS:5'-GCAGCATCAAACTGTG TAG-3'), which was expected to yield a $404 \mathrm{bp}$ amplicon. After the products were visualized on agarose gel, the remaining PCR mixture was purified with GeneJET PCR Purification Kit (Thermo Fisher). Purified PCR amplicons were sequenced with the Sanger method in Hacettepe University Nephrogenetics Laboratory. For assessing the in-frame CTNNB1 exon 3 deletions, we implemented a PCR amplification using the corresponding genomic DNA with the primers BCAT_ex2-4del_F:5'-CCAGCGTGGACAATGGCTA C-3' and BCAT_ex2-4del_R:5'-TGAGCTCGAGTCATTG CATAC-3', which was expected to yield a $931 \mathrm{bp}$ amplicon for the wild-type alleles. In case of an interstitial deletion, shorter amplicons will be generated, depending on the size of the genomic deletion.

\section{Results}

\section{Assay development to detect CTNNB1 mutation in FFPE tissues by immunohistochemistry}

Our hypothesis for this study was that the cancer-associated mutated forms of $\beta$-catenin that might be present in CRCs and other neoplastic lesions would be readily detected with 
Fig. 1 Development of an immunohistochemical assay for the detection of mutant $\beta$-catenin using the HCT 116 cell line. A hematoxylin and eosin (H\&E)stained section is shown (a). The serine 45 CTNNB1 mutation harbored by the HCT116 cell line is confirmed via Sanger sequencing (b). Strong cytoplasmic/nuclear accumulation of $\beta$-catenin with the antibody against total $\beta$ catenin is seen along with the lack of staining with the anti$\mathrm{ABC}$ (active $\beta$-catenin) antibody via immunohistochemistry (c, d). Scale bars, $100 \mu \mathrm{m}$
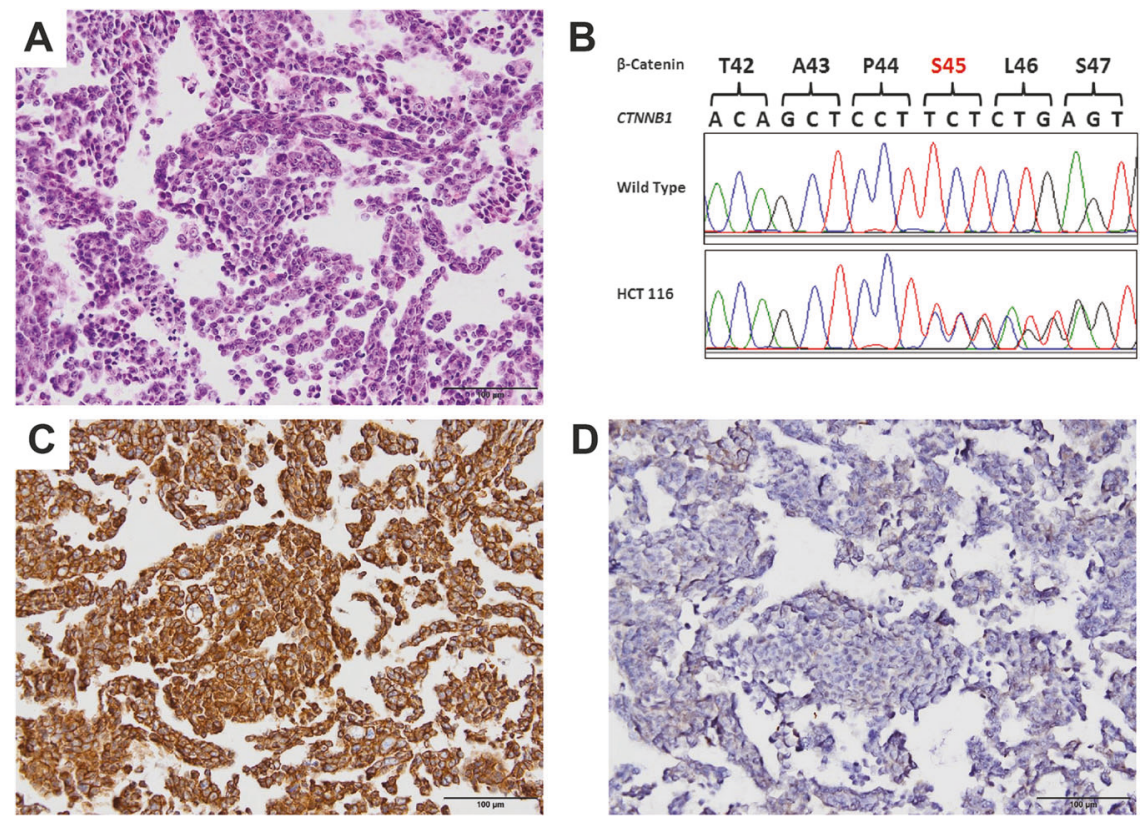

an antibody against the body of the $\beta$-catenin protein. However, oncogenic mutant $\beta$-catenin proteins harboring defects that directly affect the $\beta$-catenin amino-terminal sequences would not be detected by the anti-active $\beta$-catenin (anti-ABC) antibody. The anti-ABC recognizes unphosphorylated amino acids in the critical amino-terminal region of $\beta$-catenin (amino acids 29-49, including serine 45 that is initially phosphorylated by casein kinase-1 (CK1) and threonine 41 , serine 37 , and serine 33 that are then phosphorylated by GSK3 $\beta$ ). This is the region of $\beta$-catenin that is specifically affected in the oncogenic missense or amino-terminal-deleted mutant forms of $\beta$-catenin found in cancers. To address this hypothesis, we have performed two immunohistochemical assays using consecutive sections of each specimen; first to see the cytoplasmic and/or nuclear accumulation of $\beta$-catenin, and second to see if there might be a loss of signal with the anti-ABC antibody in the same specimen.

Before testing patient tumor samples, we generated HCT 116 cell line FFPE tissue blocks and an H\&E section from the tissue block was prepared (Fig. 1a). The HCT 116 cell line has an in-frame three-base deletion in CTNNB1 [at codon serine (S) 45], resulting in a mutant form of $\beta$-catenin that is essentially constitutively unphosphorylated or hypophosphorylated, due to its inability to have the priming phosphorylation by $\mathrm{CK} 1$ at $\mathrm{S} 45$ and then the subsequent phosphorylation by GSK3 $\beta$ at the other downstream residues $[17,18]$. We confirmed the presence of the known S45 in-frame mutation by analysis of PCR amplified CTNNB1 exon 3 amplicon in HCT116 cells (Fig. 1b). Using the two monoclonal antibodies specific for $\beta$-catenin, we performed immunohistochemical staining on sections derived from
HCT 116 tissue block. As seen in Fig. 1c and d, accumulation of $\beta$-catenin was demonstrated with $\beta$-catenin antibody (BD Transduction Laboratories), but staining with the anti-ABC antibody was negative. We also validated this finding with two different antibodies raised against $\beta$-catenin for active form (Cell Signalling) and total pool (Dako) in HCT116 tissue block (Supplementary Fig. 1A-B). This simple algorithm and pattern provided promising results to further test primary tumor samples from patients.

\section{Validation of the immunohistochemical mutation detection approach for CTNNB1 in colon polyps}

We performed immunohistochemistry with the two $\beta$-catenin antibodies using consecutive sections from colon polyp TMAs. We investigated polyps, in which strong $\beta$-catenin accumulation was detectable in the cytoplasm and/or nucleus with the total $\beta$-catenin antibody, whereas the case would be negative for staining with the anti-ABC antibody. Such cases might harbor CTNNB1 mutations. We found $6(4.8 \%)$ of 126 adenomatous and serrated colon polyps examined in the TMAs had the apparent CTNNB1 mutant staining pattern (Fig. 2a-c). This data reproduced with other two set of antibodies for total and active form of $\beta$-catenin (Supplementary Fig. 1C-D). APC mutations are known to be roughly as prevalent in adenomas as CRCs [19]. In the majority of the colon polyp tissue cores, we observed the expected strong staining patterns for both total $\beta$-catenin and ABC (Fig. 2d-f). Our data suggested that the immunohistochemical approach might indeed be a straightforward method to detect CTNNBI mutations in 

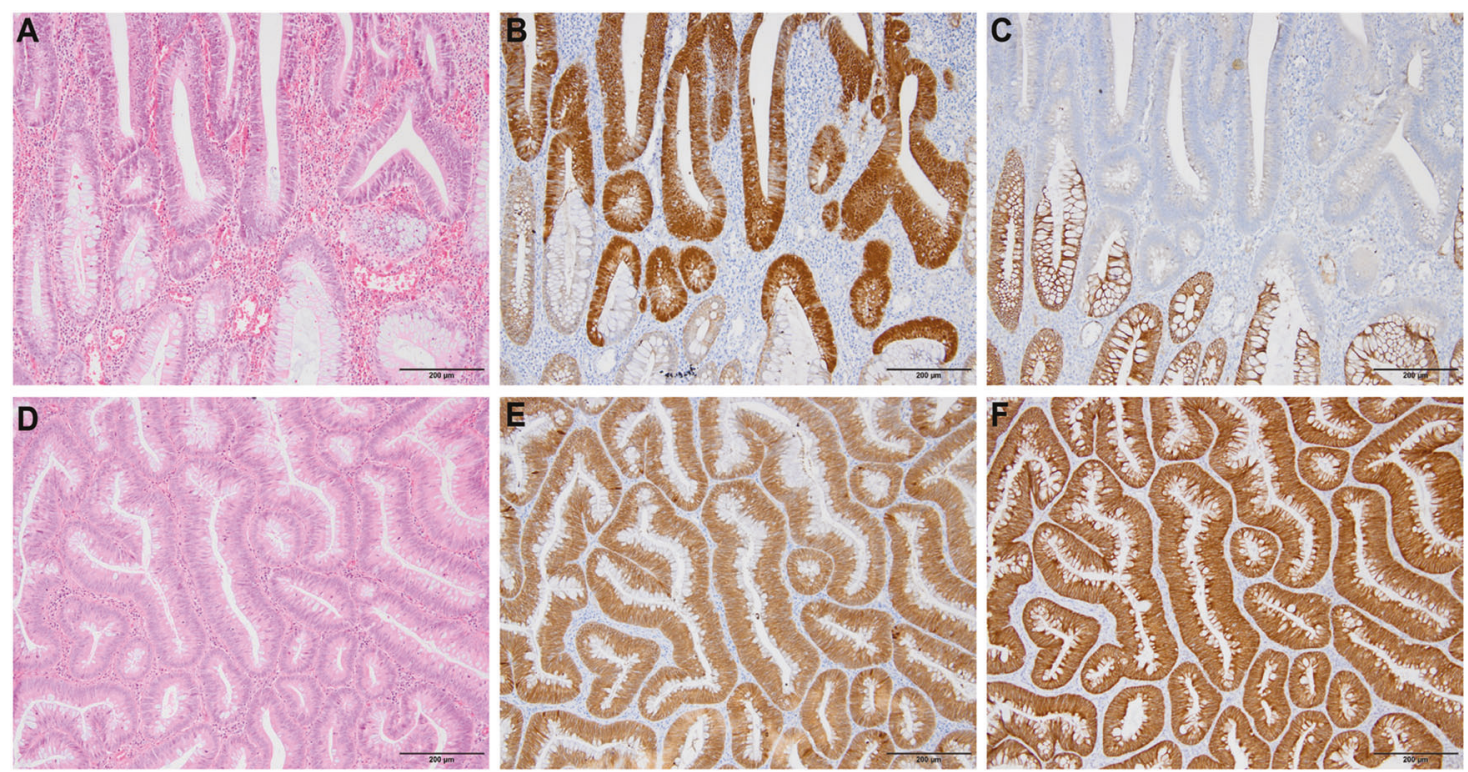

G

Adenoma case 5; CTNNB1 exon 3 deletion, CDNA sequence

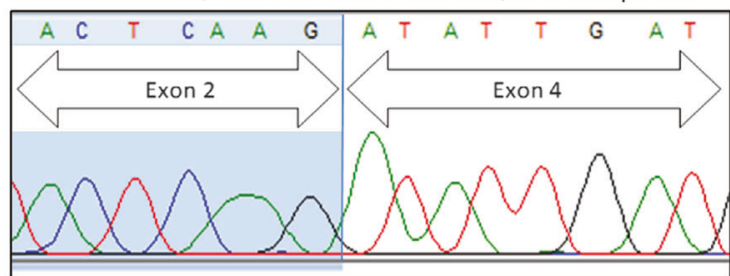

Fig. 2 The immunohistochemical approach to identify mutant $\beta$ catenin in colon adenoma TMAs. Immunohistochemical staining patterns of mutant $(\mathbf{a}-\mathbf{c})$ and wild-type $(\mathbf{d}-\mathbf{f}) \beta$-catenin (from left to

colon polyps. After finding six such adenomas via immunohistochemistry, we used DNA sequencing to assess whether CTNNBI mutations were present. A missense mutation of $\beta$-catenin codon S37 was found in one out of six cases (case number 6, Fig. 2h). The other five cases did not manifest $C T N N B 1$ point mutations. However, based on analysis of RNA isolated from the FFPE tissues of the five adenomas, we found that four of the five cases (case numbers 1, 3, 4, and 5) lacked exon 3 in the CTNNB1 transcripts present in tumor tissues and failed to obtain a product for the other one case (case number 2). CTNNB1 exon 3 encodes the $\beta$-catenin $\mathrm{N}$-terminus, including the key residues for phosphorylation regulated by GSK3 $\beta$ and CK1. We also validated the large in-frame $C T N N B 1$ heterozygous deletion of the genomic exon 3 loci by PCR amplification with BCAT_ex2-4del_F and R primers of corresponding adenomas. The normal expected PCR amplicon size was $931 \mathrm{bp}$, but we observed truncated PCR products for four adenoma cases (case numbers 1, 2, 3, and 4) and failed to obtain a product for the other one case (case number 5). Detailed clinicopathological information about mutant colon polyps is given in Table 1 .
$\mathrm{H}$

Adenoma case 6; CTNNB1 exon 3 S37 coding region mutation

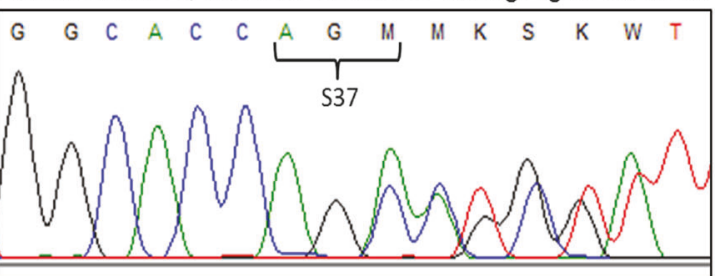

right: $\mathrm{H} \& \mathrm{E}$, total $\beta$-catenin, active $\beta$-catenin/ABC). CTNNB1 exon 3 deletion in RT-PCR (g) and point mutation in CTNNB1 S37 (h) are seen. Scale bars, $200 \mu \mathrm{m}$

\section{Detection of altered/mutant form of $\beta$-catenin in CRC TMAs}

We next assessed the immunohistochemical CTNNB1 mutation detection approach on CRCs. Using four TMAs constituting 202 CRC specimens, we undertook staining with both the $\beta$-catenin antibodies. The $\beta$-catenin-stained slides and corresponding $\mathrm{H} \& \mathrm{E}$ sections were scanned with Olympus VS 120 Digital Pathology System. Cores were evaluated for $\beta$-catenin accumulation with both antibodies (Supplementary Fig. 2). Out of the 202 CRCs samples studied, only 181 cases could be compared for the difference in staining due to technical problems where some of the arrayed tissue cores were lost from one or more of the TMAs. We found that $35(19.3 \%)$ of 181 cases showed the presumptive altered/mutant CTNNB1 immunohistochemical staining pattern for $\beta$-catenin (Fig. 3). Of these 35 cases, 10 cases were randomly selected for further molecular testing for CTNNBI exon 3 mutations, with the same approach which was used to study the colon adenomas. Of the 10 cases studied, we found one case with a heterozygous missense mutation in exon 3 of $C T N N B 1$ gene at codon P52 
Table 1 Clinicopathological features of colon adenomas with CTNNB1 mutation

\begin{tabular}{lllllll}
\hline Case number & Histologic type of polyp & $\begin{array}{l}\text { Patient's age } \\
\text { (years) }\end{array}$ & Sex & Location of polyp & Polyp diameter & Mutation of CTNNB1 \\
\hline 1 & 71 & Male & Hepatic flexure & $1.6 \mathrm{~cm}$ & $C T N N B 1$ exon 3 deletion \\
2 & $\begin{array}{l}\text { Traditional serrated } \\
\text { Tabnoma }\end{array}$ & 70 & Male & Rectosigmoid region & $2.4 \mathrm{~cm}$ & $C T N N B 1$ exon 3 deletion \\
3 & Tubulovillous adenoma & 69 & Male & Rectum & $1.5 \mathrm{~cm}$ & $C T N N B 1$ exon 3 deletion \\
4 & Tubulovillous adenoma & 69 & Female & Transverse colon & $1.2 \mathrm{~cm}$ & $C T N N B 1$ exon 3 deletion \\
5 & Tubular adenoma & 51 & Male & Sigmoid colon & $1.2 \mathrm{~cm}$ & $C T N N B 1$ exon 3 deletion \\
6 & Tubular adenoma & 33 & Female & Sigmoid colon & $2.5 \mathrm{~cm}$ & Mutation in exon 3 affects \\
& Tubular adenoma & & & & & S37 \\
\hline
\end{tabular}
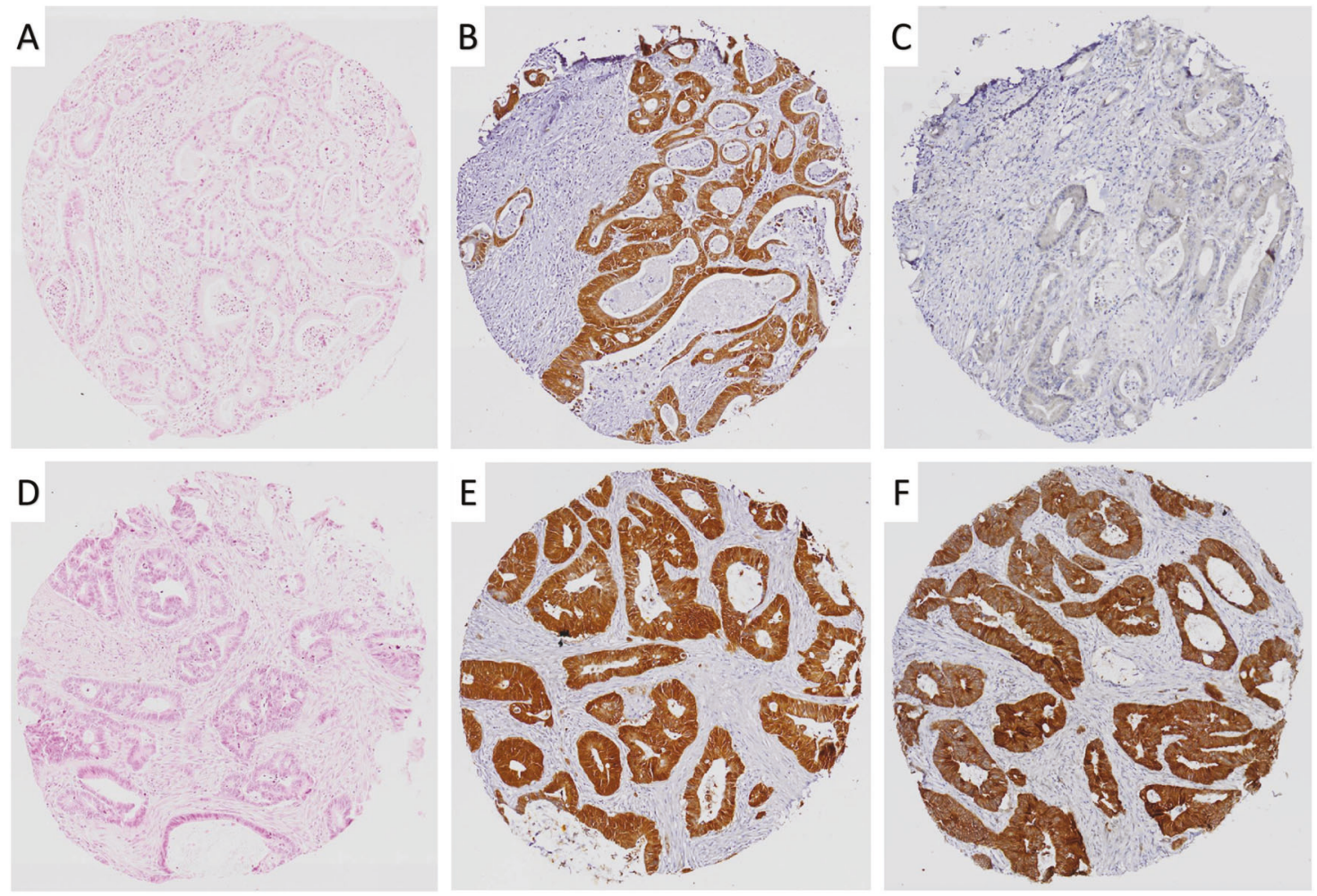

Fig. 3 Staining patterns encountered in CRC tissue microarrays for $\beta$-catenin; mutant (a-c), and wild type (d-f) (from left to right: H\&E, $\beta$-catenin, active $\beta$-catenin/ABC). Cases with altered/mutant $\beta$-catenin fail to stain with the $\mathrm{ABC}$ (active $\beta$-catenin) antibody. $1 \mathrm{~mm}$ TMA cores

(CDS mutation; c.155C > T, AA mutation; p.P52L), two cases had CTNNB1 exon 3 deletion in cDNA samples of corresponding CRCs, and one case had large genomic interstitial deletion of $C T N N B 1$. In the remaining six cases, no evidence of $C T N N B 1$ exon 3 molecular defects was found. Pertinent clinicopathological features of the CRCs evaluated are summarized in Table 2. The degree of tumor differentiation, gender, stage, metastatic status, tumor location, mismatch repair protein status did not exhibit a statistically significant association with $\beta$-catenin altered/mutant status (Pearson chi-square, data not shown). Cases with altered/mutant $\beta$-catenin seemed to show poor survival than those with intact $\beta$-catenin, but the association did not reach statistical significance (Log-Rank, $p=0.206$, Supplementary Fig. 3). We also tested $\beta$-catenin mutant staining pattern in additional 40 CRC clinical cases by immunohistochemical staining of whole tissue sections. Consecutive sections stained with active and total $\beta$-catenin antibodies, and evaluated for altered/mutant staining pattern. We found two clinical cases of CRC $(2 / 40,5 \%)$ in this analysis. 
Table 2 Clinicopathological features of CRCs

\begin{tabular}{|c|c|}
\hline Characteristic & $n(\%)$ \\
\hline Age (years), median (range) & $62(19-93)$ \\
\hline \multicolumn{2}{|l|}{ Sex } \\
\hline Female & $62(34.3)$ \\
\hline Male & $119(65.7)$ \\
\hline \multicolumn{2}{|l|}{ Tumor location } \\
\hline Right colon & $64(35.4)$ \\
\hline Left colon & $113(62.4)$ \\
\hline Other/undetermined & $3(1.7)$ \\
\hline Synchronous tumor & $1(0.6)$ \\
\hline Total & 181 \\
\hline $\operatorname{Size}^{\mathrm{a}}(\mathrm{cm})$, mean (range) & $5.523(1.7-15)$ \\
\hline \multicolumn{2}{|l|}{ Stage $^{b}$} \\
\hline I & $13(8.4)$ \\
\hline II & $49(31.8)$ \\
\hline III & $44(28.6)$ \\
\hline IV & $48(31.2)$ \\
\hline \multicolumn{2}{|l|}{ Status } \\
\hline Alive & $133(73.5)$ \\
\hline Deceased & $48(26.5)$ \\
\hline \multicolumn{2}{|c|}{ Follow-up duration $^{\mathrm{c}}$ (days) median (range) } \\
\hline \multicolumn{2}{|l|}{$994(5-4978)$} \\
\hline \multicolumn{2}{|c|}{$\beta$-catenin mutation status via IHC } \\
\hline Present & $35(19.33)$ \\
\hline Absent & $146(80.66)$ \\
\hline
\end{tabular}

${ }^{a}$ Known for $177 / 181$ cases $(97.79 \%)$

${ }^{\mathrm{b}}$ Known for $154 / 181$ cases $(85.08 \%)$

${ }^{\mathrm{c}}$ Known for $177 / 181$ cases $(97.79 \%)$

\section{Significance of immunohistochemical testing for $\beta$ - catenin alterations in other tumors known to harbor Wnt pathway defects}

Wnt pathway dysregulation is seen in a number of different tumor types besides CRCs, and CTNNB1 exon 3 missense mutations are commonly seen in certain types, desmoid fibromatosis [20], pilomatrixoma [21], and solid pseudopapillary tumor of pancreas [22]. Hence, we tested the concurrent anti- $\beta$-catenin and anti-ABC antibody staining approach in these types of tumors. We studied 6 cases of desmoid fibromatosis, 9 cases of pilomatrixoma, and 13 cases of solid pseudopapillary tumor of pancreas. We observed the altered/mutant staining pattern for $\beta$-catenin in these tumors were $6 / 6$ (100\%) for desmoid fibromatosis, 5/9 (56\%) for pilomatrixomas, and 6/13 (46\%) for the solid pseudopapillary tumor of pancreas (Fig. 4). We did further mutational analysis of exon 3 of $C T N N B 1$ in these tumors with altered/mutant $\beta$-catenin immunohistochemical staining pattern. We found CTNNB1 exon 3 mutations in five cases $(n=6,83.3 \%)$ in desmoid fibromatosis, two cases $(n=4,50 \%)$ in pilomatrixomas, and five cases $(n=5$, $100 \%$ ) in solid pseudopapillary neoplasm of pancreas. Mutation details in non-colonic tumors were summarized in Supplementary Table. The anti-ABC monoclonal antibody, clone $8 \mathrm{E} 7$ also cross-reacts with a nuclear antigen that is not $\beta$-catenin [23]. We also observed this staining pattern in some of our samples (Supplementary Fig. 4). This nuclear antigen produces a signal in some spindle cells; however, this finding does not interfere with our inference of the mutational status of tumors by $\beta$-catenin immunohistochemistry.

\section{Discussion}

Surgical pathology specimens are routinely processed to prepare FFPE blocks for diagnosis and other applications. Immunohistochemistry is well-defined approach in many pathology laboratories, and $\beta$-catenin immunohistochemistry is a commonly used method to identify tumors with Wnt pathway dysregulation. Detection of positive $\beta$-catenin staining in the cytoplasm and/or nucleus of cells is thought to reflect Wnt pathway activation. Among the gene defects that can lead to constitutive activation of the $\mathrm{Wnt} / \beta$-catenin pathway in neoplasia is mutational inactivation of the $A P C$ tumor suppressor gene, which is observed in about $80 \%$ of CRCs $[1,2,5]$. A presumptive pathological mutation in the $C T N N B 1$ gene affecting the $\beta$-catenin amino-terminal region was first described in the human gastric cell line HSC-39 more than two decades ago [24]. Missense and inframe deletion mutations affecting exon 3 of the CTNNBI gene and leading to stabilization of $\beta$-catenin have been reported in colon cancer and melanoma cell lines [17, 18, $25,26]$. A CTNNB1 mutation was found to be less prevalent in larger colon adenomas than in smaller ones, although the focus of the work was only oncogenic defects that left $C T N N B 1$ exon 3 intact, such as missense mutations [27]. In another study, interstitial deletions of $C T N N B 1$ exon 3 were found in CRCs, and missense mutations in exon 3 were not found [28]. In Japanese patients, deletion of exon 3 of CTNNB1 could be identified in 3 out of 100 colon adenomas [29]. In our series, we found evidence for CTNNB1 mutations in 6 of 126 colon polyps. All six polyps with CTNNB1 mutations were adenomas. One of the adenomas had a missense mutation and the other five mutations were in-frame genomic deletions of exon 3.

In CRCs, CTNNB1 mutations leading to stabilization of $\beta$-catenin vary in frequency in different studies. In the 2012 Cancer Genome Atlas reference analysis, the CTNNB1 mutation rate was $5 \%$ in the non-hypermutated CRCs, and $7 \%$ in the hypermutated CRCs [2]. In a recent study of metastatic CRCs, Wnt pathway alterations were seen in 

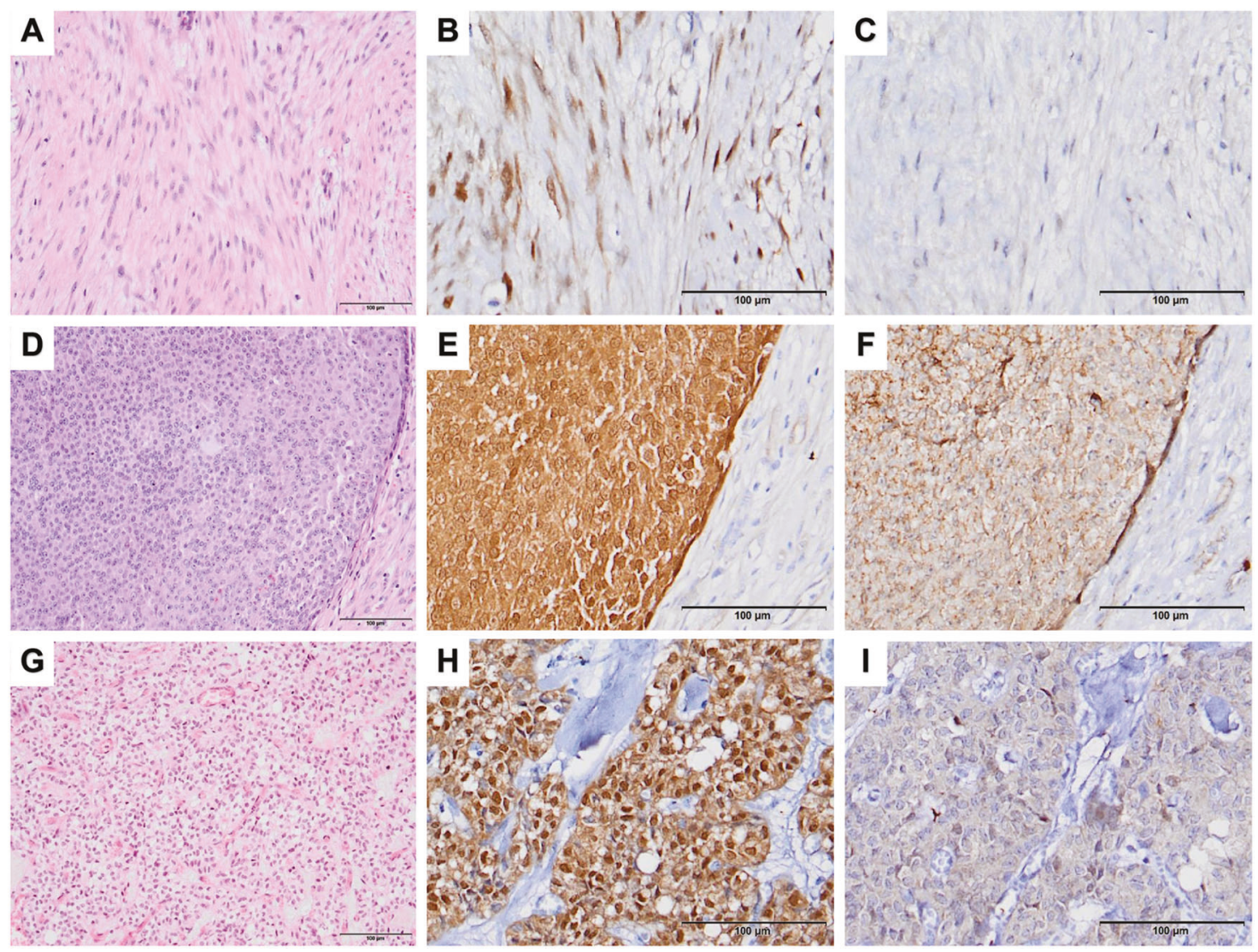

Fig. $4 \mathrm{H} \& \mathrm{E}$ and $\beta$-catenin immunohistochemical staining patterns in desmoid fibromatosis $(\mathbf{a}-\mathbf{c})$, pilomatrixoma of skin $(\mathbf{d}-\mathbf{f})$, and solid pseudopapillary tumor of pancreas $(\mathbf{g}-\mathbf{i})$. H\&E stains on the left and positive $\beta$-catenin immunohistochemistry assays in the middle. These tumors are known to harbor altered/mutant $\beta$-catenin. Altered/mutant $\beta$-catenin fails to stain with the $\mathrm{ABC}$ (active $\beta$-catenin) antibody (right). Scale bars, $100 \mu \mathrm{m}$ almost $96 \%$ cases, with in-frame deletions in $C T N N B 1$ exon 3 identified in $8 \%$ of metastatic CRCs [30]. In our CRC series, we found that $19 \%$ of CRCs showed an altered/ mutant $\beta$-catenin immunohistochemical staining pattern in TMA cases. The factors that might account for the increased prevalence of the altered/mutant $\beta$-catenin staining pattern in our series of CRCs include potential technical and biological issues. For instance, one technical issue might be the limitations inherent in the interpretation of staining patterns in small portions of primary tumor material represented in the two tissue cores from each of the primary specimen present in a given TMA. TMAs were used in this study to facilitate analysis of a large collection of tumor specimens at reduced costs, and it remains to be determined whether analysis of larger portions of primary tumor material for altered/mutant $\beta$-catenin staining might yield a lower frequency of altered/mutant $\beta$-catenin calls than the $19 \%$ achieved here. In immunohistochemical analysis for whole tissue sections in 40 different CRC clinical cases, we found 2 CRCs with altered/mutant staining pattern for $\beta$-catenin out of 40 clinical cases (5\%). This result also support that
TMAs with small tissue cores had limited value for our immunohistochemical mutational assessment for $\beta$-catenin in FFPE tissues. In terms of biological mechanisms other than $C T N B B 1$ exon 3 defects that might contribute to the presence of high levels of $\beta$-catenin in the cytoplasm and nucleus that does not react with the anti-ABC antibody, it is possible in some of these cancers that $\beta$-catenin might be phosphorylated by GSK3 $\beta$ at the multiple residues in $\beta$ catenin's amino-terminal domain - and thus not detected by the anti-ABC antibody, but $\beta$-catenin might not be efficiently targeted for ubiquitination and/or degradation due to mutations in the protein complexes that regulate $\beta$-catenin's ubiquitination and proteasomal degradation. Consistent with this notion, mutations affecting $\beta \operatorname{TrCP}$ have been reported in some cancer types and are associated with nuclear staining for $\beta$-catenin $[31,32]$. Epigenetic silencing of AXIN2 and $\beta \operatorname{TrCP}$ has been implicated in the stabilization of $\beta$-catenin in cancers, in the absence of APC mutations [33].

Besides using the immunohistochemical approach to detect altered/mutant $\beta$-catenin in colon tumors, we also 
tested the approach in selected human tumors which are known to have $\beta$-catenin alterations like desmoid fibromatosis [20, 34], pilomatrixoma of skin [21], and solid pseudopapillary tumor of pancreas [22]. Our results clearly demonstrated the efficacy of our approach to detect $C T N N B 1$ alteration in these other tumors.

In conclusion, we propose that a simple immunohistochemical approach might be useful for detecting altered/ mutant $\beta$-catenin in FFPE tumor samples from pathology archival material. While it has been known for a long time that cytoplasmic and nuclear accumulation of $\beta$-catenin is a key feature of various Wnt-activated tumors arising in different organ systems, we propose that our approach will allow further dissection of the intrinsic nature of mutated $\beta$ catenin, from extrinsic mechanisms. It will also uncover tumors that can be studied in depth and provide new insights into key factors and mechanisms besides APC, $\mathrm{AXIN} 1 / 2$, and $\beta \operatorname{TrCP}$ that regulate $\beta$-catenin levels and localization in normal and cancer conditions.

Acknowledgements This work was funded by The Scientific and Technological Research Council of Turkey (TUBITAK) under the 1001 program with the project numbers SBAG-109S475 and SBAG$113 \mathrm{~S} 985$.

\section{Compliance with ethical standards}

Conflict of interest The authors declare that they have no conflict of interest.

\section{References}

1. Fearon ER. Molecular genetics of colorectal cancer. Annu. Rev Pathol. 2011;6:479-507.

2. The Cancer Genome Atlas N. Comprehensive molecular characterization of human colon and rectal cancer. Nature. 2012;487:330-7.

3. Polakis P. The many ways of Wnt in cancer. Curr Opin Genet Dev. 2007;17:45-51.

4. MacDonald BT, Tamai $\mathrm{K}, \mathrm{He} \mathrm{X}$. Wnt/beta-catenin signaling: components, mechanisms, and diseases. Dev Cell. 2009;17:9-26.

5. Polakis P. Wnt signaling and cancer. Genes Dev. 2000;14:1837-51.

6. Dar MS, Singh P, Mir RA, Dar MJ. Beta-catenin N-terminal domain: an enigmatic region prone to cancer causing mutations. Mutat Res. 2017;773:122-33.

7. Sharma A, Sen JM. Molecular basis for the tissue specificity of $\beta$ catenin oncogenesis. Oncogene. 2013;32:1901-9.

8. Feng Y, Sakamoto N, Wu R, Liu J-Y, Wiese A, Green ME, et al. Tissue-specific effects of reduced $\beta$-catenin expression on adenomatous polyposis coli mutation-instigated tumorigenesis in mouse colon and ovarian epithelium. PLoS Genet. 2015;11:e1005638.

9. Bakker ER, Hoekstra E, Franken PF, Helvensteijn W, van Deurzen $\mathrm{CH}$, van Veelen $\mathrm{W}$, et al. $\beta$-Catenin signaling dosage dictates tissue-specific tumor predisposition in Apc-driven cancer. Oncogene. 2012;32:4579-85.

10. Guo Z, Lloyd RV. Use of monoclonal antibodies to detect specific mutations in formalin-fixed, paraffin-embedded tissue sections. Hum Pathol. 2016;53:168-77.
11. Ritterhouse LL, Barletta JA. BRAF V600E mutation-specific antibody: a review. Semin Diagn Pathol. 2015;32:400-8.

12. Piton N, Borrini F, Bolognese A, Lamy A, Sabourin J-C. KRAS and BRAF mutation detection: is immunohistochemistry a possible alternative to molecular biology in colorectal cancer? Gastroenterol Res Pract. 2015;2015:753903.

13. Hwang L-A, Phang BH, Liew OW, Iqbal J, Koh XH, Koh XY, et al. Monoclonal antibodies against specific p53 hotspot mutants as potential tools for precision medicine. Cell Rep. 2018;22:299-312.

14. Staal FJT, Noort M van, Strous GJ, Clevers HC. Wnt signals are transmitted through $\mathrm{N}$-terminally dephosphorylated $\beta$-catenin. EMBO Rep. 2002;3:63-8.

15. van Noort M, Meeldijk J, van der Zee R, Destree O, Clevers H. Wnt signaling controls the phosphorylation status of beta-catenin. J Biol Chem. 2002;277:17901-5.

16. Banerjee SK, Makdisi WF, Weston AP, Mitchell SM, Campbell DR. Microwave-based DNA extraction from paraffin-embedded tissue for PCR amplification. Biotechniques. 1995;18:768-70. $772-3$.

17. Ilyas M, Tomlinson IP, Rowan A, Pignatelli M, Bodmer WF. Beta-catenin mutations in cell lines established from human colorectal cancers. Proc Natl Acad Sci USA. 1997;94:10330-4.

18. Morin PJ, Sparks AB, Korinek V, Barker N, Clevers H, Vogelstein $\mathrm{B}$, et al. Activation of beta-catenin-Tcf signaling in colon cancer by mutations in beta-catenin or APC. Science. 1997;275:1787-90.

19. Powell SM, Zilz N, Beazer-Barclay Y, Bryan TM, Hamilton SR, Thibodeau SN, et al. APC mutations occur early during colorectal tumorigenesis. Nature. 1992;359:235-7.

20. Lazar AJF, Tuvin D, Hajibashi S, Habeeb S, Bolshakov S, Mayordomo-Aranda E, et al. Specific mutations in the betacatenin gene (CTNNB1) correlate with local recurrence in sporadic desmoid tumors. Am J Pathol. 2008;173:1518-27.

21. Chan EF, Gat U, McNiff JM, Fuchs E. A common human skin tumour is caused by activating mutations in beta-catenin. Nat Genet. 1999;21:410-3.

22. Tanaka Y, Kato K, Notohara K, Hojo H, Ijiri R, Miyake T, et al. Frequent beta-catenin mutation and cytoplasmic/nuclear accumulation in pancreatic solid-pseudopapillary neoplasm. Cancer Res. 2001;61:8401-4.

23. Maher MT, Flozak AS, Hartsell AM, Russell S, Beri R, Peled ON, et al. Issues associated with assessing nuclear localization of $\mathrm{N}$ terminally unphosphorylated beta-catenin with monoclonal antibody 8E7. Biol Direct. 2009;4:5.

24. Kawanishi J, Kato J, Sasaki K, Fujii S, Watanabe N, Niitsu Y. Loss of E-cadherin-dependent cell-cell adhesion due to mutation of the beta-catenin gene in a human cancer cell line, HSC-39. Mol Cell Biol. 1995;15:1175-81.

25. Robbins PF, El-Gamil M, Li YF, Kawakami Y, Loftus D, Appella $\mathrm{E}$, et al. A mutated beta-catenin gene encodes a melanoma-specific antigen recognized by tumor infiltrating lymphocytes. J Exp Med. 1996;183:1185-92.

26. Rubinfeld B, Robbins P, El-Gamil M, Albert I, Porfiri E, Polakis P. Stabilization of beta-catenin by genetic defects in melanoma cell lines. Science. 1997;275:1790-2.

27. Samowitz WS, Powers MD, Spirio LN, Nollet F, van Roy F, Slattery ML. Beta-catenin mutations are more frequent in small colorectal adenomas than in larger adenomas and invasive carcinomas. Cancer Res. 1999;59:1442-4.

28. Iwao K, Nakamori S, Kameyama M, Imaoka S, Kinoshita M, Fukui $\mathrm{T}$, et al. Activation of the beta-catenin gene by interstitial deletions involving exon 3 in primary colorectal carcinomas without adenomatous polyposis coli mutations. Cancer Res. 1998;58:1021-6. 
29. Murata M, Iwao K, Miyoshi Y, Nagasawa Y, Yabu M, Himeno S, et al. Activation of the beta-catenin gene by interstitial deletions involving exon 3 as an early event in colorectal tumorigenesis. Cancer Lett. 2000;159:73-8.

30. Yaeger R, Chatila WK, Lipsyc MD, Hechtman JF, Cercek A, Sanchez-Vega F, et al. Clinical sequencing defines the genomic landscape of metastatic colorectal cancer. Cancer Cell. 2018;33:125-36.e123.

31. Kim CJ, Song JH, Cho YG, Kim YS, Kim SY, Nam SW, et al. Somatic mutations of the beta-TrCP gene in gastric cancer. APMIS. 2007;115:127-33.
32. Gerstein AV, Almeida TA, Zhao G, Chess E, Shih I-M, Buhler K, et al. APC/CTNNB1 (beta-catenin) pathway alterations in human prostate cancers. Genes Chromosomes Cancer. 2002;34:9-16.

33. Tseng R-C, Lin R-K, Wen C-K, Tseng C, Hsu H-S, Hsu W-H, et al. Epigenetic silencing of AXIN2/betaTrCP and deregulation of p53-mediated control lead to wild-type beta-catenin nuclear accumulation in lung tumorigenesis. Oncogene. 2008;27:4488-96.

34. Huss S, Nehles J, Binot E, Wardelmann E, Mittler J, Kleine MA, et al. $\beta$-catenin (CTNNB1) mutations and clinicopathological features of mesenteric desmoid-type fibromatosis. Histopathology. 2013;62:294-304. 